\title{
Concept of information laser: from quantum theory to behavioural dynamics
}

\author{
Andrei Khrennikov ${ }^{1,2, a}$, Zeno Toffano ${ }^{3}$, and François Dubois ${ }^{4}$ \\ ${ }^{1}$ National Research University for Information Technology, Mechanics and Optics \\ (ITMO), Laboratory of Quantum Cognition and Intelligent Systems, St. Petersburg, \\ 197101, Russia \\ ${ }^{2}$ International Center for Mathematical Modeling in Physics and Cognitive Sciences \\ Linnaeus University, Växjö 35195, Sweden \\ ${ }^{3}$ Laboratoire des Signaux et Systèmes, L2S (UMR8506 - CNRS), CentraleSupélec, \\ U. Paris-Saclay, Gif-sur-Yvette, France \\ ${ }^{4}$ CNAM-Paris, Department of Mathematics, University Paris-Sud, Orsay, France
}

Received 5 March 2018 / Received in final form 29 June 2018 Published online 13 February 2019

\begin{abstract}
Recently, the methods of quantum theory, especially quantum information, started to be widely applied outside of physics: in cognitive, social sciences, economics, finance, decision making and biology. We propose a quantum-like model: the "information laser". The basic assumption is the discrete structure of state spaces related to the quantization of information. The information field acts in the form of indistinguishable quanta of "social energy" analogue to photons. The massive flow of information acts as a laser pump. In this framework, an information selection process by agents under constant pressure of massive repeated information leads to collective "resonance" effects in analogy with laser cavity and stimulated emission. In order to make operational parallels between physical lasers and the information laser we identify the essential features of laser operation. An application to the analysis of recent disruptive social events (colour revolutions) is discussed. Social analogues to the laser are also considered through the model of Echo Chambers induced by the Internet and Adam Smith's invisible hand.
\end{abstract}

\section{Introduction}

We propose an approach concerning stimulated generation of coherent social actions, used for the description and analysis of the outburst of dramatic social events (revolutions) introduced in reference [1]. Because of the complexity of these social phenomena, one of the goals of this concept is to extract the pertinent features and identify them with the corresponding laser concepts.

The formalism of quantum mechanics is now widely explored to describe biological, cognitive, psychological, and socio-political phenomena. It is currently applied outside of physics in cognitive-social sciences, economics and biology to model

a e-mail: Andrei.Khrennikov@lnu.se 
context-sensitive systems: it constitutes the quantum-like paradigm (Khrennikov), which provides a consistent probabilistic picture of observations performed for systems exhibiting (statistically) quantum-like features. The information laser is based on the quantization of information describing stimulated generation of coherent social actions [2] characterized by indistinguishability, a central issue of the application of quantum theory to social science. Quantum indistinguishability was already an issue in the proposed Fractaquantum hypothesis [3], which applies to all indivisible scales in nature; regardless of their size, it addresses the incompatibility of quantum indistinguishability with macroscopic structures. Recently, the quantum formalism has been used naturally for logical propositional reasoning in Eigenlogic, extending the possibilities of classical logic by giving a fuzzy and multivalued approach using quantum observables [4]. Such kind of logic matches very well quantum-like modelling of cognitive, social and political phenomena.

In this paper, after reminding the different models, we will build a correspondence of social behaviours with the actual effects observed in physical lasers. Our aim is to identify the essential features of laser operation and the associated key physical parameters and connect them (at the formal operational level) with key social parameters in the process of stimulated generation of coherent social actions.

It has to be outlined that at the very beginning of laser research, parallels have been made between laser behaviour and other disciplines, considering the laser concept as a general principle with potential applications outside physics, for example in the Synergetics approach by Haken [5], where the focus was put on the laser phase transition and non-linear dynamics analogy and in the works on the laser statistical aspects by Lax [6]. On one hand, ideas of Haken and Lax support our modelling of information lasers and their applications to social processes. On the other hand, and in contrast with them, we do not only discuss analogies. By establishing the correspondence between basic features and parameters of quantum physical processes and quantum-like structures of social processes, we specify the basic constraints on social "gain media" and information fields leading to stimulated generation of coherent social actions.

We have to stress here that at the present stage, this nascent concept has to be considered more as a metaphor than a working model. It will be useful as a guideline for social analogies. On the other hand, a full quantum model of the physical laser is still an open question. Our quantum-like approach could in the future be extended to a precise quantitative model, in association with more refined quantum models for the main issue, which is the notion of social energy and the quantification of the energy spectrum of a human agent. This quantification can be undertaken in the future, of course, in cooperation with experts from cognitive and social sciences (see Sect. A.3 for a brief review on applications of the concept of social energy in humanities, economics, biological evolution, climate change, economics, and industrial dynamics).

Recently, the possibility to apply the mathematical formalism of quantum theory outside of physics was actively explored in cognitive science, psychology, economics, finance, social and political sciences, decision making, game theory, and computer science (e.g. quantum-like models for information retrieval), as thoroughly reported in recent monographs [6-13] and representative papers [14-23]. These are all applications of the quantum-like approach. They should be sharply distinguished from the attempts to reduce human cognition and hence behaviour to genuine quantum physical processes in the brain (see, e.g. Refs. [24,25]). In the quantum-like approach, humans are considered "black boxes" processing information using the laws of quantum information and probability, the quantum formalism is thus treated as an operational formalism and quantum mechanics is endowed with the information interpretation in the spirit of Zeilinger and coworkers [26], D'Ariano [27], Plotnisky [28], and Fuchs and Schack [29]. This quantum-like approach was originated by one of the coauthors of this paper [14] and was then successfully applied to a variety of problems. The main 
motivation to apply the quantum formalism for the prediction of observation outcome probabilities is the widely recognized impossibility to model human behaviour with classical probabilistic models (such as the von Neumann-Morgenstern [30] expected utility theory, the subjective expected utility theory, or the prospect theory).

Decision-making theories based on classical probability theory have generated many paradoxes (starting with the seminal Allais and Ellsberg paradoxes). Attempting to resolve these paradoxes led to various modifications of the theory, but each new modification suffered of new paradoxes. Tversky and Kahenman [31,32] and other researchers in psychology and economics demonstrated numerous situations where the classical probability reference persistently departed from actual human behaviour. Tversky and Kahenman have promoted the heuristic approach as an alternative to probabilistic modelling of decision making. However, recently it has also been demonstrated that the basic classical decision theory paradoxes disappear in a quantum-like decision-making model [22]. Moreover, quantum-like approaches have been actively used to model the basic effects of cognitive psychology and decision making such as the order, conjunction, and disjunction effects [8]. These successful applications of quantum formalism outside of physics are very supportive of our "social laser" concept.

We also emphasize that the majority of the "users" of quantum-like approaches belong to the fields of humanities, economics, and computer science. We expect that the present paper will be a useful guide for these experts. At the same time, we hope that it will also attract the attention of physicists, especially experts in quantum information and foundations as well as laser physics, to seek novel application possibilities of the quantum formalism.

The paper is organized as follows:

In Section 2, we will explain the principal laser features, starting in Section 2.1 from the quantum basics relating the interplay of statistics of atoms and photons in a laser. Then, the laser field equations are discussed in Section 2.2. They are associated with the different behaviours depending on laser threshold. In Section 2.3, the important features of coherence and photon correlation are discussed. Laser linewidth determining the degree of coherence is then analysed in Section 2.4.

In Section 3, a system model for the laser is proposed. In Section 3.1, the role of laser cavity is discussed. In Section 3.2, the coefficients for spontaneous and stimulated emission are related to the gain coefficient in the expression of the laser intensity. Laser oscillation conditions due to the interplay of cavity and gain are given in Section 3.3. In Section 3.4, the important role of spontaneous emission entering the laser field for the intensity and the phase is analysed pointing out its effect on laser linewidth.

In Section 4, the discussion is shifted towards the general concept of information laser by making the correspondence between the physical and the information models. In Section 4.1, a minimal set of key parameters are identified. In Section 4.2, the problem of indistinguishability of the laser excitations is discussed in the perspective of heuristic decision making. In Section 4.3, a general correspondence is proposed.

Section 5 presents the Internet Echo Chambers as a social analogue of a laser cavity. Adam Smith's invisible hand concept is also discussed as an analogue of a thermodynamic open system as is the case of the laser.

An appendix is devoted to some macroscopic heuristic pictures of a general information laser: Section A.1 the cavity, Section A.2 the role of laser threshold and Section A.3 the social energy.

\section{Quantum features of a laser}

On a fundamental level, the quantum features at the origin of the laser behaviour remain an open question. A full quantum analysis of a laser [33], leads to involved 
quantum diffusion Fokker-Planck equations using quantum operators. But the concept of a laser has been evolving with the better understanding of laser physics. At the beginning, a laser was understood as a source of an intense, sharp beam, but it was soon realized that the high intensity was not the only attribute of a laser; one must also consider its coherence, or photon statistics, that constitutes the most fundamental distinction of laser light from light of a usual lamp. The coherence of a laser is more specifically described by the first- and second-order correlation functions (see hereafter) which are very popular in quantum optics. One of the points of practical importance is to determine when a light-emitting device has exceeded the so-called threshold and becomes an actual laser.

\subsection{Particle statistics in a laser}

What are the quantum features that underlie laser action? This is a complex matter because of the interaction of different quantum systems in a laser. Photons which constitute the emitted light interact with energy levels inside the active media and are also coupled to the cavity.

Photons obey Planck's statistics (Bose-Einstein statistics for massless particles such as photons) and are represented in quantum field theory by the Bosonic creation operator $a^{\dagger}$ and annihilation operator $a$, satisfying the commutation relation $\left[a^{\dagger}, a\right]=$ $a^{\dagger} \cdot a-a \cdot a^{\dagger}=1$. The quantum observable for this system is the photon number operator given by

$$
n=a^{\dagger} \cdot a
$$

corresponding for a laser to the number of photons in the considered mode.

Radiative transitions in the active media are between two energy levels: $E_{1}$, the lower level, and $E_{2}$, the upper level. This constitutes a quantum two-level system and can be treated in quantum mechanics as a fermion-mediated transition obeying the Pauli exclusion principle on each level. Also here one can introduce creation and annihilation operators, respectively, for the two levels. So, for an energy level $E_{i}$, one defines the operators $c_{i}^{\dagger}$ and $c_{i}$, satisfying now an anti-commutation relation: $\left\{c_{i}^{\dagger}, c_{i}\right\}=c_{i}^{\dagger} \cdot c_{i}+c_{i} \cdot c_{i}^{\dagger}=1$.

A full quantum analysis using quantum density operators can be undertaken, and several approaches have been proposed [33], the result being complex formulation which is essentially a Fokker-Planck type equation involving quantum operators. By all these efforts, some simplifying guidelines can be given, quoting Haken: "the basic quantum mechanical laser equations are strongly reminiscent of the semiclassical laser equations. Indeed, the quantum mechanical laser equations look the same as the semiclassical laser equations with the exception of an additional term which represents fluctuating forces."

\subsection{Equations for the laser photon field}

Here we describe the basic models characterizing laser emission. The following equations are obtained by a semiclassical approach for a single-mode laser behaviour. One defines two equations for the normalized fluctuations of the photon field $b$ and atomic field $\alpha$ :

$$
\delta \dot{b}=\left(-i \omega-\Delta \omega_{c}\right) \delta b-i \chi \delta \alpha, \quad \delta \dot{\alpha}=\left(-i \varpi-\Delta \omega_{a}\right) \delta \alpha+i \chi d_{0} \delta b
$$


where

- $\omega$ is the cavity resonance angular frequency;

- $\varpi$ is the angular frequency corresponding to the laser transition energy: $\hbar \varpi=$ $h \nu=E_{2}-E_{1}$, the laser frequency $\nu$ is $\varpi / 2 \pi$;

$-\Delta \omega_{c}=\tau_{p}^{-1}$, the cavity bandwidth and $\tau_{p}$ the photon field relaxation time in the cavity;

- $\Delta \omega_{a}=\tau_{a}^{-1}$, the atomic bandwidth and $\tau_{a}$ is the atomic relaxation time;

- $d_{0}$ is the unsaturated normalized inversion parameter of the atom population proportional to the difference of population between the two atomic levels (see next section) $d_{0} \propto \Delta N=\left(N_{2}-N_{1}\right)$;

- $\chi$ is the field-atom coupling coefficient.

Considering the general coherent field amplitudes $b=B e^{-i \Omega t}$ and $\alpha=A e^{-i \Omega t}$, one can solve the two preceding equations and identify the coefficients. The formulation of the field frequency $\Omega$, including all contributions, is given by an expression comprising all the contributions listed above:

$$
\Omega=\left(\varpi \Delta \omega_{c}+\omega \Delta \omega_{a}\right) /\left(\Delta \omega_{c}+\Delta \omega_{a}\right) .
$$

This expression shows that in general the frequency of the laser field $\Omega$ does not coincide with the frequency of the cavity. But most of the times, for a well-designed laser, we have $\Omega \sim \omega \sim \varpi$ and the differences are not significant.

In the field equations given above, the amplitude $b$ and its conjugate $b^{*}$ have their counterpart in the quantum bosonic operators $a$ and $a^{\dagger}$. The important parameter is of course the photon number $n=|b|^{2}$, which in a fluctuation analysis will correspond to the average number of photons.

The atomic field $\alpha$ is the counterpart of the quantum fermion operators $c$ and $c^{\dagger}$. Inversion can be treated quantum mechanically using the fermion operators corresponding to the two atomic energy levels $E_{1}$ and $E_{2}$ leading to the corresponding inversion operator [33]:

$$
\hat{d}=c_{2}^{\dagger} c_{2}-c_{1}^{\dagger} c_{1}
$$

There is a further simplification which consists in the adiabatic elimination of the atomic variations, assuming a selective cavity where $\Delta \omega_{c} \ll \Delta \omega_{a}$. This leads to a simplified photon field equation with a Langevin driving fluctuation noise source $F(t) \propto \delta(t):$

$$
\dot{b}=\beta\left(\Delta-|b|^{2}\right) b+F(t)
$$

where the coefficients are explicitly: $\beta \sim\left[4 \Delta \omega_{c}^{2} /\left(\Delta \omega_{a} d_{t h r}\right)\right]$ and $\Delta \sim d_{0}-d_{t h r}$. This last term is the driving parameter, which can be considered as an order parameter of the laser phase transition. $d_{0}$ is the steady state inversion and corresponds to the mean value $\langle\hat{d}\rangle$ of the quantum atomic inversion operator defined above.

The field equation shows a Van der Pol oscillator form, its interpretation is simple: the nonlinear term in the brackets represents a potential function and is effective only when $\Delta>0$, that is when the inversion $d_{0}>d_{t h r}$ is above the laser threshold. The mean value of the photon field $\bar{b}=\sqrt{\left\langle b(t)^{*} b\left(t^{\prime}\right)\right\rangle}$ can be easily derived considering the fluctuation force, which gives 
$-\bar{b}_{<}=0$ below threshold

$-\bar{b}_{>}=\sqrt{n} \neq 0$ above threshold

where $n$ is the average photon number. This represents, above threshold, a Poisson statistical distribution for the photons with the standard deviation satisfying: $\Delta n=$ $\sqrt{\left\langle n^{2}\right\rangle-\langle n\rangle^{2}}=\sqrt{n}$. This last expression shows the important result that the laser effect takes place above threshold with a stable intensity.

One can then derive the expression of the laser threshold condition for the inversion parameter given by $d_{0}>\left(\Delta \omega_{c} \Delta \omega_{a}\right) /\left(\chi^{2}\right)=d_{t h r}$. A comment about this last expression is that positive inversion, $d_{0}>0$, is not sufficient alone for laser action, one must also overcome the losses of the cavity and of the material medium, which are globally represented by the term $d_{t h r}$. The region corresponding to $0<d_{0}<d_{t h r}$ is the amplifying region where one observes amplified spontaneous emission.

\subsection{Coherence and correlation of the laser photon field}

The coherence properties of a laser beam are obtained by averaging the fluctuating quantities. An important difference between incoherent light and laser light can be highlighted by higher order correlation functions. One introduces the first-order correlation function, $g^{(1)}$, and the second-order correlation functions, $g^{(2)}$, as follows:

$$
g^{(1)}(\tau)=\left\langle b(t)^{*} b(t+\tau)\right\rangle, g^{(2)}(\tau)=\left\langle b(t)^{*} b(t+\tau)^{*} b(t) b(t+\tau)\right\rangle .
$$

For a laser, the first-order correlation function well above threshold, $g^{(1)}(\tau)$, can be expressed as a function of the average photon number $n$ and of the phase fluctuations:

$$
g_{\text {laser }}^{(1)}(\tau) \sim \operatorname{nexp}\left(-\Delta \omega_{\varphi} \tau\right), \quad\left\langle(\varphi(\tau)-\varphi(0))^{2}\right\rangle \approx 2 \Delta \omega_{\varphi} \tau
$$

where $\Delta \omega_{\varphi}$ is the linewidth of the laser field. The laser behaviour above threshold consists in stable intensity and all the fluctuations are due to the phase. The laser coherence is characterized by its linewidth. One speaks of phase diffusion and its purest form for a laser corresponds to a Lorentzian lineshape, in this case the phase can be considered a Gaussian random variable.

The second-order correlation function $g^{(2)}$ plays a very important role in the field of quantum optics that was first studied in the mid-1950s [35]. The quantum theory of light can predict non-classical values of $g^{(2)}$ that are completely impossible for conventional light waves. The signature of the photon nature of light can have a three-fold classification [34], in which the light is described as bunched, coherent or anti-bunched. This is summarized for the three cases by

- bunched light: $g^{(2)}(0) \geq g^{(2)}(\tau) \geq 1$

- coherent light: $g^{(2)}(\tau)=g^{(2)}(0)=1$

- anti-bunched light: $g^{(2)}(0) \leq g^{(2)}(\tau) \leq 1$.

Anti-bunched light is only possible in the photon interpretation and is thus another clear signature of the quantum nature of light, which corresponds to sub-Poissonian statistics for photons $(\Delta n<n)$. Anti-bunched light has practical applications in single-photon sources, which are fundamental building blocks in experiments for quantum information. The Hong-Ou-Mandel effect is a two-photon 
interference effect in quantum optics which was demonstrated in 1987 [36]: when the photons are identical, they will extinguish each other, giving $g^{(2)}(0)=0$, a clear signature of anti-bunching.

More specifically, for a laser the second-order correlation function $g^{(2)}(\tau)$ increases below threshold from the completely incoherent case, due to spontaneous emission, where $g_{s p}^{(2)}=2$ up to a maximum and then decreases to the limit $g_{\text {las }}^{(2)}=1$ well above threshold. So, for a laser well above threshold, the photon statistics variance $(\Delta n)^{2}$ follows the Poisson's behaviour equal to its mean value $\bar{n}_{L}$ :

$$
(\Delta n)^{2}=\bar{n}_{L}
$$

The situation below threshold is different and will be discussed in more detail in the next section. In general, for less coherent sources than lasers, one has superPoissonian light with $(\Delta n)^{2}>\bar{n}$. The most incoherent situation for photon statistics is thermal blackbody radiation characterized by the Bose-Einstein Planck photon distribution $\bar{n}_{T}$ at temperature $T$. In this case, it is easily shown [34] that

$$
\bar{n}_{T}=1 /(\exp (h \nu / k T)-1), \quad(\Delta n)^{2}=\bar{n}_{T}+\bar{n}_{T}^{2}=\bar{n}_{T}\left(1+\bar{n}_{T}\right) .
$$

The first term, $\bar{n}_{T}$, in the expression of the variance $(\Delta n)^{2}$ corresponds to the particle nature of light, as in the Poissonian distribution, while the second term, $\bar{n}_{T}^{2}$, originates from the thermal fluctuations of the energy of the electromagnetic radiation. This fact was already noticed by Einstein when he introduced the model for the emission and absorption mechanisms involving photons with thermal energy density $\rho_{T}=\left(8 \pi h \nu^{3} / c^{3}\right) \bar{n}_{T}$, which has led to the definition of the Einstein's coefficients presented hereafter. Summarizing, the first term is a quantum contribution and the second a classical one.

\subsection{Laser linewidth}

It is interesting to observe, as mentioned before, that the problem of the quantum origin of laser light remains still today an open question. Recently, in the context of photon correlation for quantum information, an interesting discussion and auto-critics has been led by Wiseman [37] then followed by a controversial reply by Elsäßer [38]. One important conclusion from these discussions is that there exists a crucial property of laser light: a laser must have stable intensity. More precisely, above threshold intensity, fluctuations become insignificant and only phase noise characterizes the statistics of a laser beam. The simplest quasi-classical model for this behaviour is a constant diffusion Fokker-Planck equation for the phase probability distribution $p(\phi)$ :

$$
d p(\phi) / d t=\Delta \omega / 2 \quad\left(d^{2} p(\phi) / d \phi^{2}\right)
$$

where $\Delta \omega=2 \pi \Delta \nu$ is the laser linewidth.

The difference of behaviour below and above threshold has been experimentally verified. A complete analysis based on experiments on semiconductor lasers using a Fokker-Planck model has been proposed by one of the authors in reference [39], showing a phase transition behaviour.

In the following, we will present the expressions for the laser linewidth using the fundamental parameters presented above. The average number of photons $n$ can be 
related to the output power $P$ using

$$
P=2 \Delta \omega_{c} n h \nu
$$

for a laser with cavity bandwidth $\Delta \omega_{c}$ (see discussion on cavities). Then, the linewidth below and above threshold can be simply expressed as follows:

$$
\Delta \omega_{<}=2 h \nu \Delta \omega_{c}^{2} P^{-1}, \quad \Delta \omega_{>}=h \nu \Delta \omega_{c}^{2} P^{-1} .
$$

Below threshold, the expression of the linewidth scales differently with a factor of 2 when compared with the one above threshold. This is due to the additional amplitude fluctuations below threshold. These expressions are called the Schawlow-Townes laws [40] for the laser linewidth showing the laser threshold phase transition behaviour. The spectral linewidth for a laser below and above threshold can be put in a very simple form as a function of the photon number $n$, the cavity characteristics $\Delta \omega_{c}$ and the second-order correlation function $g^{(2)}(0)$ (as discussed earlier, this quantity varies from the value 2 to the value 1 when passing the threshold $[33,34,37,39]$ ):

$$
\Delta \omega=g^{(2)}(0) \Delta \omega_{c} n^{-1}
$$

Similar expressions will be derived in the following section using a system-like approach.

\section{Operational properties of laser emission: a system approach}

Since we hope that this paper will be comprehensible for researchers from the fields of psychology, sociology, management and political science, we start with the brief recollection of the history of laser. The invention of the laser was one of the main technological breakthroughs of quantum physics. The theoretical basis for the laser invention was implicitly present in the works of Einstein on stimulated absorption and emission in 1917. Also in 1939, the Soviet physicist Fabrikant considered the theoretical possibility of stimulated amplification of microwaves. But only in the 1950s, Townes in cooperation with Gordon and Zeiger designed and realized in the laboratory the first microwave amplifier. Nowadays such devices are known as masers. Townes's maser was incapable of continuous output. In 1955, Prokhorov and Basov suggested optical pumping in a multilevel system as a method for obtaining the population inversion, later a key method of laser pumping.

According to Haken in reference [33], the best-known and typical properties of a laser are high intensity, high directionality, spectral purity, coherence and laser output as ultrashort pulses. Coherence is a broad concept that encompasses several laser features such as spectral purity but also polarization and directionality.

A first question is what makes the laser so special and why is it different from, say, an ordinary light bulb? What is sometimes confusing is that when considering the acronym LASER (lightwave amplification of stimulated emission of radiation), one associates this device with an amplifier, but this is only part of the story. Operating lasers are actually light generators similar to electronic oscillators where the amplifier is submitted to positive feedback. Feedback is achieved in a laser by using an optical cavity surrounding the active media. Generally, it is a Fabry-Pérot cavity made of up of partially reflecting mirrors as shown in Figure 1.

It is interesting to examine how the transition from non-coherent emission (e.g. ordinary lamp) to that of a laser takes place. Again according to Haken [33], "If we 
pump the laser only weakly ... the light field consists of entirely uncorrelated individual wave tracks... When we increase the pump power beyond a certain threshold, an entirely new behaviour of laser light emerges. The behaviour corresponds to an extremely long wave track." It has also to be noticed that the laser threshold transition is continuous [39]. Just below threshold, the device is in the filtered amplified spontaneous emission regime often denoted as superradiant.

From an operational point of view, one has to distinguish two situations:

1. A laser as a resonant amplifier: when increasing the pump, the total bandwidth (spectral width) of the light output reduces. One has amplified spontaneous emission.

2. A laser as a generator: when pumping reaches the laser threshold, then the gain "clamps" and all the supplementary pumping energy is "transferred" to the stimulated emission. The optical power increases linearly with the pump power up to very high levels only limited by the heating of the device. The linewidth decreases as the inverse of the power, giving a highly coherent beam.

\subsection{The role of the laser cavity}

From an historical point of view, the breakthrough of Charles Townes was that of introducing the cavity in the laser. He started with the maser in 1953, where he used microwaves corresponding to the ammonia molecule transition [39], and the original idea was to amplify most efficiently microwave signals using cavities.

In order to fully grasp the behaviour of a physical laser, we will explain here the role of the cavity. A cavity is an optical resonator which is a passive component and so does not by itself amplify energy; it confines it and transfers the energy to selected resonance modes. In order to amplify, one must place the pumped active media inside the cavity. Also in this way a resonant amplifier can become an oscillator for sufficient pumping.

The most common type of lasers uses a Fabry-Pérot cavity. Light bounces back and forth between the mirrors, passing through the gain medium and being amplified each time (see Fig. 1). Typically, one of the two mirrors, the output coupler, is partially transparent, some of the light escapes through this mirror.

Cavities are characterized by their bandwidth $\Delta \omega=2 \pi \Delta \nu$, which is related to the "quality factor" $Q$ by

$$
\omega / \Delta \omega=Q=\omega(\mathcal{E} / P)
$$

with $\omega=2 \pi \nu$, where $\nu$ is the frequency, $\varepsilon$ is the energy (unit: joule) stored in the cavity and $P$ the power lost from the cavity. Because $Q$ can be very high, the cavity bandwidth $\Delta \nu_{c}$ is typically several orders of magnitude less than the atomic gain bandwidth $\Delta \nu_{g}$ of the laser material around the two-level transition frequency $\nu$ satisfying

$$
E_{2}-E_{1}=h \nu=\hbar \varpi
$$

where $E_{2}$ is the upper energy level and $E_{1}$ is the lower energy level and $\varpi$ is the angular atomic transition frequency.

The cavity quality factor $Q$ defined above is one of the important system parameters for the laser [41]. All kinds of losses and also the gains, giving a negative contribution, can be included to get a global quality factor linked to the several important laser parameters. 


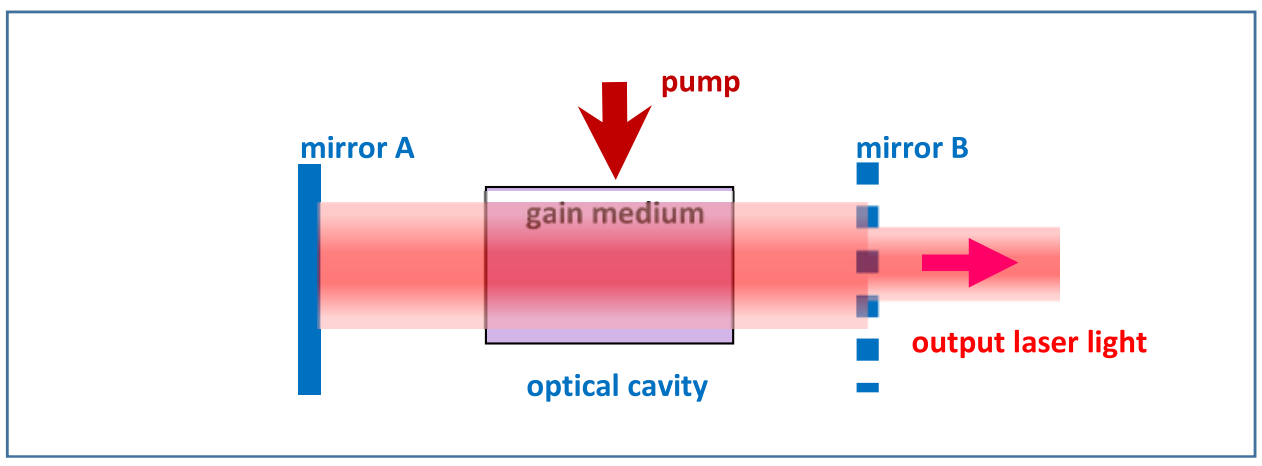

Fig. 1. Fabry-Pérot laser cavity with partially reflecting mirror B.

\subsection{Spontaneous, stimulated emission and gain}

Laser operations involve important parameters. First, the Einstein coefficients for spontaneous emission $\mathrm{A}$, absorption $B_{12}$ and stimulated emission $B_{21}$.

The spontaneous emission rate coefficient $A$ is the inverse of the spontaneous lifetime $\tau_{s p}$ :

$$
A=1 / \tau_{s p}
$$

The induced effects of stimulated emission and absorption are characterized, respectively, by the coefficients $B_{21}$ and $B_{12}$. Here, in a simplified approach, one does not consider atomic degeneracy; this gives the equality of the induced coefficients $B_{21}=B_{12}=B$. The spontaneous and induced coefficients are related by

$$
A / B=8 \pi h \nu^{3} / c^{3}
$$

where $c$ is the speed of light, $h$ is Planck's constant, and $\nu$ is the frequency.

Rate equations describe the evolution of the atomic populations and photons in the laser process. Considering the intensity of a laser beam $I(\nu)$ as the number of energy quanta $h \nu$ (the photons) produced per second and per unit surface, the rate equation for the photon intensity is given by

$$
d I(\nu) / d t=h \nu\left(N_{2}-N_{1}\right) B g(\nu) I(\nu)=c \gamma I(\nu)
$$

where $N_{1}$ and $N_{2}$ are the population densities (unit: inverse of a volume) of the energy levels $E_{1}$ and $E_{2}$ and $g(\nu)$ is the normalized gain profile characterized by the gain bandwidth $\Delta \nu_{g}$. The solution of the above equation leads, when converting time $t$ to space variation $z$ by the photon velocity $c$, to an exponential increase or decrease of the laser intensity along the cavity depending on the sign of the gain coefficient $\gamma$. Looking at equation (18), this corresponds to the sign of the inversion term $\Delta N=N_{2}-N_{1}$ in front of the intensity $I(\nu)$.

\subsection{Laser oscillation conditions}

Considering a cavity with length $L$ and a coupling mirror reflection factor $r$, the intensity after one reflection will be multiplied by the loss factor $r e^{-\alpha_{c} L}$. So, the 
condition for sustained power in the cavity is

$$
\gamma L>\alpha_{c} L=-\log r
$$

This condition imposes a minimum gain coefficient, the threshold gain $\gamma_{t h}=\alpha_{c}$, which compensates exactly the cavity loss. Because generally the reflection factor $\mathrm{r}$ is very close to 1 , we have $\alpha_{c} L=-\log r \simeq(1-r)$, this last term is related to the quality factor of the cavity $Q_{c}$. Using the definition given in equation (14), one has the expression $Q_{c}=2 \pi \nu \mathrm{Vol} / \mathrm{cS}(1-r)=2 \pi \nu L / c(1-r) \simeq 2 \pi \nu / c \alpha_{c}$, where $(1-r)$ corresponds to the fraction of the energy lost by the cavity through one facet, $\mathrm{Vol}$ is the cavity volume and $\mathrm{S}$ the cross-section surface, the cavity length is $L=\mathrm{Vol} / \mathrm{S}$.

The cavity bandwidth is given by $\Delta \nu_{c}=\nu / Q_{c}$ corresponding to the bandwidth of a cavity mode which decreases when the reflection factor $r$ approaches $1(100 \%$ of reflection). Another interesting parameter is the photon lifetime in the cavity $\tau_{p}$, which can be expressed as a function of the cavity quality factor $Q_{c}$ or also as a function of the cavity bandwidth $\Delta \nu_{c}$ :

$$
\tau_{p}=Q_{c} / 2 \pi \nu=1 / 2 \pi \Delta \nu_{c}=1 / c \alpha_{c}
$$

Frequencies inside the cavity must verify the constructive interference condition after a round trip in the cavity - they represent the modes of the cavity. The frequency of a mode $\nu_{m}$ labelled by the number $m$ and the frequency spacing between modes $\delta \nu$ are, respectively, given by

$$
\nu_{m}=m c / 2 L, \quad \delta \nu=\nu_{m}-\nu_{m-1}=c / 2 L .
$$

One can also estimate the number of allowed modes $M$ in the cavity, which depends on the cavity volume $\mathrm{Vol}$ and on the gain bandwidth $\Delta \nu_{g}$. The mode density $\rho_{m}$ (per unit volume) is then given as follows:

$$
\rho_{m}=M / V o l=8 \pi \nu^{2} \Delta \nu_{g} / c^{3} .
$$

\subsection{Spontaneous emission, coherence and linewidth}

During the amplification process when the linewidth is reduced, one can describe this effect by considering the noise introduced by the spontaneous emission coefficient $A$. The two principal noise sources are the spontaneous emission noise and the thermal noise due to the photons in the cavity (thermodynamic heat bath).

Because energy is maintained in the cavity on an average time $\tau_{p}$, the contribution to the spontaneous energy per mode will be

$$
\varepsilon_{s p}=h \nu\left(N_{2} / \rho_{m}\right)\left(\tau_{s p} / \tau_{p}\right) .
$$

Thermal noise is characterized by the photon Planck distribution in the cavity, giving the thermal energy contribution per mode: $\varepsilon_{T}=h \nu /\left(e^{h \nu / k T}-1\right) \simeq h \nu e^{-h \nu / k T}$. Considering that for lasers optical frequencies verify $h \nu \gg k T$ at operating temperatures; even for a low value of inversion, one can assume that $\varepsilon_{s p} \gg \epsilon_{T}$, meaning that the spontaneous emission noise is predominant in a laser.

The total energy stored in the cavity $\mathcal{E}$ verifies the following equation, including spontaneous emission:

$$
d \mathcal{E} / d t=2 \pi \nu \mathcal{E} / Q_{c}=\operatorname{Vol}\left[h \nu B g(\nu)\left(N_{2}-N_{1}\right) \mathcal{E}+h \nu A g(\nu) N_{2}\right]
$$




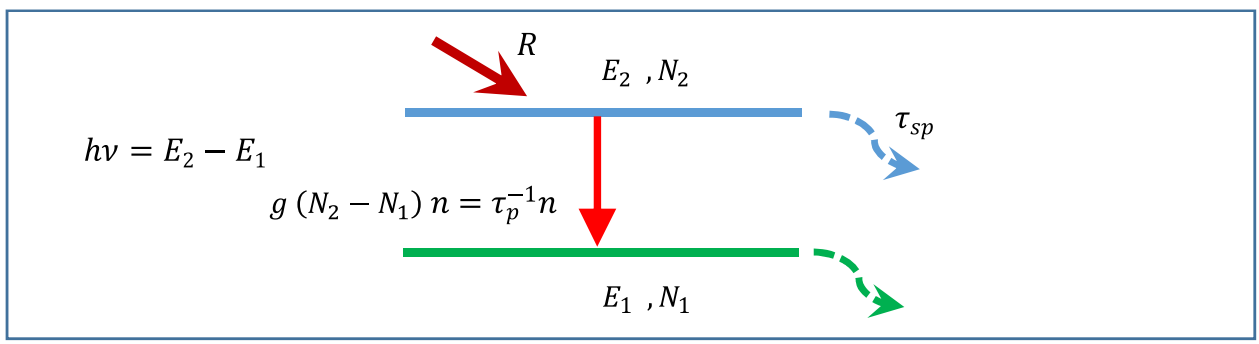

Fig. 2. Laser mechanism in the two-level system characterized by the system parameters used in the rate equations for pump rate $R$, inversion $\Delta N=N_{2}-N_{1}$ and power $P$ developed in Appendix A.

This equation can be transformed using the above expressions as $2 \pi \nu \mathcal{E}\left[Q_{c}^{-1}-(c \gamma / 2 \pi \nu)\right]=2 \pi \nu Q_{c}^{-1}\left[N_{2} /\left(N_{2}-N_{1}\right)\right] h \nu$, this last term is the spontaneous emission contribution. One can now estimate the actual linewidth $\Delta \nu$, including the amplification effects:

$$
\Delta \nu / \nu=Q_{c}^{-1}-(c \gamma / 2 \pi \nu)=Q_{c}^{-1}-Q_{a}^{-1}
$$

One introduces here the quality factor $Q_{a}=2 \pi \nu c / \gamma$ due to amplification, which is responsible for reducing linewidth (minus sign). One can also express these quantities as a function of the laser output power $P$ using equation (14), which leads to the following expression for the linewidth (Schawlow-Townes law) as a function of $P$, of $\Delta \nu_{c}$, and of the inversion fraction $n_{s p}=\left[N_{2} /\left(N_{2}-N_{1}\right)\right]: \Delta \nu=2 \pi h \nu n_{s p}\left(\Delta \nu_{c}^{2} / P\right)$.

In an ideal laser operating at high power, the inversion fraction tends to $n_{s p} \sim 1$ when $N_{2} \gg N_{1}$, which leads a very simplified expression for the linewidth:

$$
\Delta \omega=2 \pi \Delta \nu=P^{-1}\left(h \nu / \tau_{p}^{2}\right) .
$$

This expression is equivalent to the one presented earlier in the quantum approach.

\section{Correspondence between information laser and physical laser}

\subsection{Identification of key physical laser parameters}

A motivation here is to define a laser using system parameters, not relying on specific laser technology. Some laser properties are universal. A tentative correspondence can be made in Table 1.

The system parameters constitute a complete system for the rate equations (see Appendix A, Eqs. (A.1) and (A.2)) and can thus be used for describing laser behaviour. The schematic two-energy level diagram with the respective coefficients is presented in Figure 2.

The total energy is $\mathcal{E}=n h \nu=n\left(E_{2}-E_{1}\right)$. The colour of the energy is represented by the wavelength $\lambda=c / \nu$ and this is essentially brought in by selection with its correspondence to the cavity filtering characteristics.

In this way, the quantitative character of the information field is represented by the photon number $n$ only, whereas the social colour of the information is represented by the coherence characteristics that is actually induced by the filtering/selecting processes due to the cavity. All coherence characteristics can be put in this category, not only frequency $\nu$ but also other properties such as polarization or beam collimation, 
Table 1. System parameters for physical and information laser.

\begin{tabular}{|c|c|c|c|c|c|}
\hline $\begin{array}{l}\text { Laser } \\
\text { feature }\end{array}$ & $\begin{array}{l}\text { Physical } \\
\text { laser }\end{array}$ & $\begin{array}{l}\text { Internal } \\
\text { parameter }\end{array}$ & $\begin{array}{l}\text { Information } \\
\text { laser }\end{array}$ & $\begin{array}{l}\text { System } \\
\text { feature }\end{array}$ & $\begin{array}{l}\text { System } \\
\text { parameter }\end{array}$ \\
\hline Pump & Inversion & $\Delta N$ & $\begin{array}{l}\text { Exciting } \\
\text { information } \\
\text { field }\end{array}$ & Pump rate & $R$ \\
\hline Amplification & $\begin{array}{l}\text { Atomic gain } \\
\text { Medium }\end{array}$ & $g, \tau_{s p}$ & $\begin{array}{l}\text { Behavioural } \\
\text { agent gain } \\
\text { medium }\end{array}$ & $\begin{array}{l}\text { Gain band- } \\
\text { width }\end{array}$ & $\Delta v_{g}$ \\
\hline Frequency & $\begin{array}{l}\text { Photon } \\
\text { energy }\end{array}$ & $E_{2}-E_{1}$ & $\begin{array}{l}\text { Social } \\
\text { colour }\end{array}$ & Mode colour & $u$ \\
\hline Lifetime & $\begin{array}{l}\text { Optical } \\
\text { cavity }\end{array}$ & $L, r$ & $\begin{array}{l}\text { Repetition/ } \\
\text { selection of } \\
\text { information }\end{array}$ & $\begin{array}{l}\text { Coherence } \\
\text { lifetime }\end{array}$ & $\tau_{p}$ \\
\hline Power & $\begin{array}{l}\text { Photon } \\
\text { number }\end{array}$ & $n$ & $\begin{array}{l}\text { Rush of } \\
\text { social } \\
\text { energy }\end{array}$ & Power & $P$ \\
\hline Coherence & $\begin{array}{l}\text { Spectral } \\
\text { linewidth }\end{array}$ & $\tau_{p}, \tau_{s p}$ & $\begin{array}{l}\text { Definiteness } \\
\text { of social } \\
\text { colour }\end{array}$ & $\begin{array}{l}\text { Coherence } \\
\text { linewidth }\end{array}$ & $\Delta \nu$ \\
\hline
\end{tabular}

which in physical lasers are achieved by cavity design. One can denote a general parameter for this "colouring" property by $u$. A quantum state corresponding to an information laser can thus be characterized by a $n$-photon state in the colour mode $u$, defined by the two quantum numbers $n$ and $u$. This is actually what we could define as a social information laser mode (we assume the single-mode behaviour):

$$
\left|\Psi_{\text {info }}\right\rangle=|n, u\rangle
$$

In this model, excitations obey Bose-Einstein statistics where messages with totally different contexts can carry the same portion of social energy. The human gain medium is characterized by a discrete spectrum of the social energy and the distribution of the social energy in the individual state space follows Fermi-Dirac statistics; an individual can absorb and emit only discrete portions of the social energy (adapted to the differences between the energy levels); individuals belonging to the same gain medium have the same spectrum; the stimulated emission of social energy. The basic contribution to the information content is brought in by the laser field and can thus be associated with the photon number $n$; this quantity can be considered as independent from the energy of the atomic transition (whereas the power cannot). Many laser characteristics depend only on $n$.

\subsection{Indistinguishability of the information laser field and heuristics}

We especially emphasize the role of indistinguishability of excitations of the information field. It is the crucial feature of systems leading to quantum statistics. (In the case of the information field this corresponds to the Bose-Einstein statistics.) Indistinguishability means that agents do not perform detailed analysis of the information content of communications. They pay attention principally to the magnitude of the social energy carried by a communication and to its "colour". This is a very usual behaviour of humans. 
In decision-making theory and cognitive psychology, this sort of behaviour is known as heuristics [31,32]. The latter is based on mental shortcuts that usually involve focusing on one concrete aspect of a complex problem and ignoring the others. This approach to information processing can lead to systematic deviations from classical Boolean logic and classical Kolmogorovean probability or rational choice theory [31,32] (cf. Refs. [7-23]). Heuristic decision making can explain a variety of recent social and political mass-phenomena.

For example, people who voted in favour of "Brexit" were principally concerned by the "immigration colour", and did not consider "financial and economic colours". Also the population attracted by ISIS were focused principally on the destructive effect of Western civilization on the traditional Muslim culture and ignored the economic, cultural and scientific consequences due to ISIS.

We also remark that different social strata are "sensitive" to different "colours" of information. In the modern Western society, one can typically distinguish three main social strata: the upper class, the middle class and the lower class. Finer differentiations can be related to gender, race, ethnicity, etc.

The heuristic approach to decision making is common in humans (and animals). But as a consequence of the information overload, one of the most distinguishing features of modern society, heuristics plays also an important role in modern human practice of decision making. A human being oppressed by an overload of information coming from his working place environment, from the mass media and the Internet seeks for survival strategies by means of numerous mental shortcuts. In this way, the information overload transforms humans into perfect gain media for information lasers ("social lasers"). The high intensity of the information flow is also an essential factor for reaching the thresholds making laser functioning effective.

We also stress that, to be able to create a population of individual agents that would function as a kind of laser emitting waves of social energy, the processes of absorption and emission of social energy have to be discrete. Individual agents have to possess a discrete structure of energy levels. In this model, an agent "falling" from the upper level $E_{2}$ to the lower level $E_{1}$ cannot stop in between. In modern society, there is no place for those who do not match the discrete structure of social layers. For absorption, the situation is similar.

For a social equivalent of a laser, the basic source of social energy pumping is the information field generated by mass media and the Internet. In the "particle representation", this field can be considered as composed of quanta of information. The latter are delivered to us by a variety of information channels such as mass media, Internet, social networks, social apps, etc.

Now we turn to the human gain medium and the (quantum) information field. For simplicity, we consider the two-level structure of social energy. Each class of information communications is characterized by the social energy $n\left(E_{2}-E_{1}\right)$ carried by $n$ quanta of this field (often in the form of news or news components).

Such communications are also associated to additional characteristics someway linked to "coherence" of the type of polarization or collimation leading to what can be defined as a "social colour", represented by the general parameter $u$. For example, $u$ can represent the anti-globalism (or gay-supporting, or anti-war) label. Then, this flow generates a "coherent beam" of social actions characterized by the parameter $u$ (e.g. anti-globalism protests). For the human gain medium, such coherence of the output of the stimulated emission process can be easily explained. People in the excited state receiving quanta of information carrying the social colour $u$ emit information quanta of the same social colour.

The model must also take into account the content of an event. Similarly, in physical reality an excitation of the quantum electromagnetic field (a photon) is characterized not only by its energy but also by its internal degree of freedom, which is associated to the colour parameter $u$ and is principally a cavity effect. 
Table 2. Correspondence table of physical and social laser processes.

\begin{tabular}{|c|c|c|}
\hline Feature & Physical & Social information \\
\hline Pump & Feed power for inversion & Massive flow of information \\
\hline Cavity & Optical cavity resonator & $\begin{array}{l}\text { Repetition/selection of quanta of } \\
\text { information; Echo chamber (media) }\end{array}$ \\
\hline $\begin{array}{l}\text { Active } \\
\text { medium }\end{array}$ & Two-energy level system & Receptive individual agents \\
\hline Field quanta & Coherent photons & $\begin{array}{l}\text { Selected indistinguishable colour } \\
\text { information }\end{array}$ \\
\hline
\end{tabular}

Each class of information communications is characterized by the social energy $E$ carried by $n$ agents. Coherence corresponds to social colour sharpness (ideally one single mode $u$ ) generating a coherent beam of social actions. People in the excited state receiving quanta of information of the same social colour emit information quanta with the same social colour. Gain medium consisting of agents in the excited state and stimulated by the anti-corruption coloured information field would "radiate" a wave of anti-corruption protests. The same gain medium stimulated by some information field carrying another social colour would generate the wave of actions corresponding to this last colour.

\subsection{Correspondence and characteristics for the information (social) laser}

We dress here the principal correspondences between the physical laser and the information laser.

The principal characteristics of the information laser are

- the quantum information field;

- its excitations - quanta of information;

- gain medium represented by human agents.

The basic contribution to the information content is brought in by the laser field and can thus be associated with the photon number $n$.

The quantum information field is characterized by several features:

1. the additivity of social energy;

2. the energy transmitted to an agent by excitations of the information field is absorbed;

3. the indistinguishability of information excitations;

4. the high intensity of information pumping - to approach the threshold.

The correspondence table between physical and social laser processes is summarized in Table 2.

The information field contributing to the social energy can be associated with the photon number $n$, being independent of the frequency. The colour of the social energy is on the other hand represented by frequency and coherence and is induced by the filtering/selecting process by agents under constant pressure of massive continuous information, leading to resonance effects as in a laser cavity. The massive flow acts as a pump of indistinguishable information accumulating continuously, the result giving stimulated generation of coherent social actions. The atomic transition defines the 
two-level system energy difference, but the stabilizing character is brought in by the cavity. The colour of the social energy is induced by the filtering/selecting process by social agents under constant pressure of massive continuous information, leading to resonance as in a laser cavity.

We notice that the quantum field description of the stimulated emission is a collective effect, i.e. an atom interacts with a bunch of photons and not just with an individual photon. The crucial role is played by the Bose-Einstein statistics of the photons. In an $n$-photon state $|n, u\rangle$, for a fixed parameter $u$, the colour mode can be represented in the form of the action of the creation operator $a_{u}^{\dagger}$ on the vacuum state $|0, u\rangle$ :

$$
|n, u\rangle=\left[\left(a_{u}^{\dagger}\right)^{n} / \sqrt{n !}\right]|0, u\rangle
$$

This representation gives the possibility to find that the amplitude of the transition probability amplitude from the state $|n, u\rangle$ to the state $|n+1, u\rangle$ equals to $\sqrt{n+1}$. On the other hand, it is well known that the reverse process of absorption characterized by the transition probability amplitude from the state $|n, u\rangle$ to the state $|n-1, u\rangle$ equals to $\sqrt{n}$. Thus, in a quantum bosonic field, the increase of the number of photons leads to an increase of the probability of generation of one more photon in the same state. This constitutes one of the "quantum advantages" of laser stimulated emission, showing that the emission of a coherent photon is more probable than the absorption.

In social science and finance, the bosonic and fermionic algebras of operators were recently used in works of Bagarello and Haven [42]. It is interesting that applications outside of physics lead to the use the operator algebra mixing bosonic and fermionic features in the so-called qubit operator algebra [43] for applications to the theory of decision making. It could be an interesting issue to consider the full quantum treatment of a laser [33], which uses both the bosonic operators $a^{\dagger}$ and $a$ for the photon field and the fermionic operators used to define a laser atomic inversion operator : $\hat{d}=c_{2}^{\dagger} c_{2}-c_{1}^{\dagger} c_{1}$ as already discussed.

\section{Echo chambers and invisible hand as social analogues of a laser}

We live in the age of the information revolution, the transition from the industrial society to a new information society. The Internet and the mass media are the main driving engines of this revolution. This information society is characterized by massive flow of information generated by a variety of sources. Daily human beings "swim" in this information sea, communicating a huge variety of news about political, financial, social, ecological and cultural events throughout the world. This availability of diverse information provides new possibilities for understanding and exploring the world. This can lead to diversification of opinions and strengthening of democracy. However, at the same time, people burdened by information search ways to structure this information flow. Roughly speaking, people suffer from information diversity and overload. The Internet can help them by creating structures known as echo chambers [44]. These are closed social systems in which beliefs of people are amplified and homogenized through the feedback coupling in the information exchange process. Thus, an echo chamber plays the role of an amplifier and a sensor at the same time. This is the straightforward social equivalent of the laser cavity which amplifies the signal and at the same time performs a kind of censoring by decreasing the bandwidth and reducing the spread of polarization. 
The interesting question is the following one: "what are social analogues of cavity mirrors?" The most powerful information mirrors (generators of the feedback process) are based on Internet and Twitter posts of people inside the concrete echo chamber, e.g. a social network. These mirrors operate in well-structured social networks. Typically, such social networks are driven by special groups of people (or organizations, or companies) which structure (via feedback) the information. As an example, we can mention a multiplicity of Islamic extremist networks. Such "cavities" (in combination with excited human gain medium) can behave as information (social) lasers generating spikes of homogeneous social actions. We can point out the recent wave of Islamic militants who rushed to the ISIS state or were involved in terrorist actions in the United States, Europe and Russia. We could discuss other examples, but this subject of a future paper will be written in a less formal style. Of course, echo chambers (social laser cavities) are not reduced to the Internet-based information mirrors. Personal communications inside "physical social groups" can play the same role. But the establishment of the Internet made information mirrors much more powerful. Finally, we remark again that echo chambers are the analogues of the cavities.

Also physical cavities can serve not only as specific laser components. In the same way, echo chambers need not be associated solely with amplification. They can simply serve as homogenization devices and here we can also find interesting quantum physical analogies.

In this general analysis, one could also invoke Adam Smith's famous metaphor of the "invisible hand" [45], which reveals that when dozens or even thousands act in their own self-interest, goods and services are created that benefit consumers and producers. This teaches us that one can operate towards a collective goal, without knowing it, when one obeys to one's own liking. Smith's physical analogue for this metaphor was the mechanics of the solar system, a problem in this analogy is that the metaphor is not based on the correct physical analogue, which rather belongs to thermodynamics and statistics [46]. But it has been argued that the analogy to thermodynamics close to equilibrium holds only for closed systems, and most certainly global economy is no closed system. An analogy with our concept of information laser, that constitutes a thermodynamic open system, could perhaps enrich the metaphor for this collective effect.

\section{Conclusion}

Quoting reference [47]: "The concept of a laser has been evolving with both our ever deepening understanding of laser physics and with the progress of various laser technologies. In the very early days, a laser was understood as a source of an intense, sharp beam. Soon it was realized that the high intensity was not the most fundamental attribute of a laser. Coherence or photon statistics constitute the most fundamental distinction of the laser light from light of a regular lamp. The coherence of a laser is more specifically described by the first- and second-order correlation functions. While such correlation functions are routinely measured in the quantum optics community, most of the optoelectronics (or more generally engineering) community often relies on a few more directly measurable quantities (such as light intensity and/or linewidth) of practical importance to determine when a light emitting device has exceeded the so-called threshold and become a laser."

We want to underline that laser behaviour is due to the interplay of cavity and gain effects [48]. The cavity is not the only mechanism for picking the social colour but is responsible for the resonance effects and plays a role in filtering. The concept of coherence is central, an operational autonomous laser is an oscillator and not just an amplifier and in this configuration it can transform energy. Also it has been 
shown recently by work on microlasers [47] that even with a very poor cavity, the laser effect takes place with few photons and an "effective cavity" is brought in by nonlinear mechanisms defining a laser threshold and an oscillator type behaviour.

An application can be sought for the analysis of new socio-political phenomena such as the so-called "colour revolutions" which initiated in the territories of the former Soviet Union and in the Balkans. Recently, similar features in Western democratic systems originated from protests against government corrupted system. These phenomena are the subject of numerous studies and publications in political and social sciences (see Refs. [1,2] for discussions). An adequate theory for these dramatic sociopolitical phenomena is still lacking. In this situation, it seems motivating to explore the correspondence and to define a methodology based on the laser formalism in order to explain the outburst of these phenomena.

Finally, we remark that the modern social processes, such as sharply polarized irreconcilable ideologies, are not unprecedented. The rise of xenophobic populism with racist overtones in present-day Europe and United States (some would call it fascism) does not seem to be all that different from the earlier stages of analogous mass phenomena in the 1930s - when we had no Internet and no information overload. Nevertheless, extremely high intensity of modern information flows makes easier transition to heuristic absorption of information (i.e. ignoring the complex content of information excitations and losing the possibility to perform classical logical analysis) and the Internet provides essentially a more powerful base for Echo Chambers than meetings and demonstrations. So, it becomes much easier to generate the stimulated amplification of human actions.

\section{Appendix A: Attempting to present heuristic pictures of laser features}

\section{A.1 Cavity}

When considering coherence generated by the interplay of the laser's cavity with the gain media, it is useful to think in terms of "modes" which are the "result" of constructive interference, and are an essential contribution brought in by the cavity. A mode relates some particular solution of the degree of freedom of the electromagnetic wave: frequency, polarization (two degrees of freedom) and spatial configuration of the wave, which gives the directionality (linked in some way also to frequency because of its link to size).

It is always tricky to think of direction of propagation of photons when we are actually in a "standing wave" configuration in the cavity (one "resolves" the issue by saying that one has two counter-propagating waves). As in most issues of quantum physics, this is a question of "representations" which then turn out to be equivalent. In a laser, we are in the same "troubles", actually no one goes inside the cavity to "observe" what direction the photons propagate. What is sure is that the "output" possesses these high coherence features, polarization, directionality and monochromatic light.

A "perfect" laser should have all these features at their maximum. But because of the physical losses (intrinsic in order to get something out of a laser) and imperfections we never get optimal conditions. Bad lasers can have some or all of the coherence characteristics degraded (e.g. large spectrum, large radiation pattern, poor degree of polarization). The quality can be defined by the coherence time or length measured by the autocorrelation function. For polarization, one can define the degree of polarization by the Stokes parameters on the Stokes sphere (equivalent to the Bloch sphere, but not using pure density matrices for quantum states). 


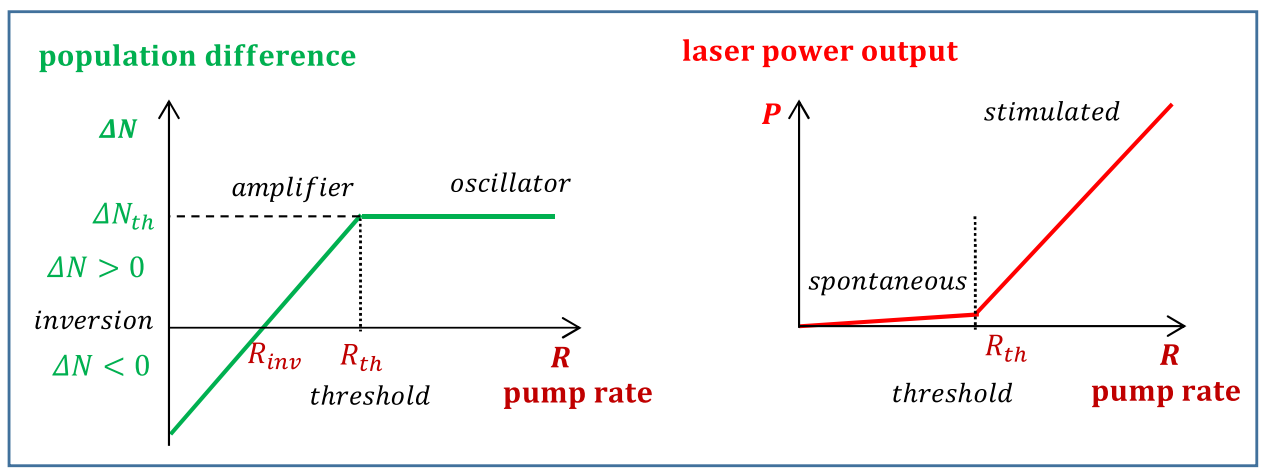

Fig. A1. Laser population difference and output power figures from the universal rateequation model

\section{A.2 Threshold}

One can be surprised by the analogies with the nonlinear behaviour of classical nonlinear oscillators, which is a topic that has been thoroughly studied from the very beginning of the development of electrical circuits. For example, the "Van der Pol" oscillator has similar nonlinear characteristics.

Nevertheless there are some "heuristic" guidelines which seem to be universal. The nonlinear gain plays a crucial role (also in electronic oscillators); it is a sort of stabilizer, it permits the system to go through the transition smoothly and then stabilizes its amplitude. A very simple way to grasp the threshold behaviour is from the "depurated" rate equations. A two rate-equation model can be used where the parameters are pump power $R$, light output power $P$ (proportional to the photon number $n$ ) and the inversion of the gain medium $\Delta N$, proportional to the population difference $N_{2}-N_{1}$.

This simple nonlinear model enables one to show the laser phase transition through the equations:

$$
\begin{aligned}
d \Delta N / d t & =R-\Delta N / \tau_{s p} \\
d P / d t & =g(P \Delta N)-P / \tau_{p} .
\end{aligned}
$$

This equation system is nonlinear, because of the product term $(P \Delta N)$ in the second equation, and leads to the characteristics shown in Figure A1. The global behaviour is of the Van der Pol oscillator type already discussed in the paper. These figures show the laser threshold phase transition. Laser modelling may be carried out at different approximation levels. Steady-state solutions of equations (A.1) and (A.2) lead to approximate expressions of laser power function of the pump rate holding below, through and above threshold [39]. These rate equations can be considered as universal for laser operation [48].

\section{A.3 Concept of social energy}

The notion of energy plays a fundamental role in physics, both classical and quantum. The concept of "social energy" is intended as a metaphor that can play a similar role in the context of socio-ecological systems. This concept is widely used not only in 
cognitive science and psychology (in close relation with the notion of mental energy), but also in social and political sciences, economics, multi-agent modelling, evolution theory and industrial dynamics (see, e.g. Refs. [49-51]). In such applications, a variety of approaches to the notion of social energy are explored. Generally, social energy is used when modelling shifts between different states of complex social systems, e.g. regime shifts in economic systems (and the energy needed to induce them or gained from them).

The following "energy-information" viewpoint is the closest to our modelling. We observe that in thermodynamics, the Helmholtz free energy includes an entropic term. Gain and losses of information in complex social systems are undeniably important variables for evaluating the available energy in a society when particular transitions take place. This entropic formalization approach of social energy is very promising and can be considered for the quantification of the social energy of system.

Another interesting approach is based on the interpretation of social energy as a potential to change. In game theory, for example, the notion of social energy is related with the notion of potential function. Grauwin et al. [49] formulated the following program "built both on statistical mechanics and on the game theoretic notion of Potential Function to introduce a rigorous generalization of the physicist's free energy for purposes of socio-economic analysis".

The notion of emotional energy (see Ref. [51]) to model the micro-macro link in social systems, including economics can also be considered.

In future research, the authors of this paper plan to establish a closer relation of the proposed model of information ("social") laser with known models using the concept of social energy.

Open Access This is an open access article distributed under the terms of the Creative Commons Attribution License (http://creativecommons.org/licenses/by/4.0), which permits unrestricted use, distribution, and reproduction in any medium, provided the original work is properly cited.

\section{References}

1. A. Khrennikov, Philos. Trans. R. Soc. 374, 20150094 (2016)

2. A. Khrennikov, Entropy 17, 6969 (2015)

3. F. Dubois, Res-Systemica 5, 55 (2006)

4. F. Dubois, Z. Toffano, Eigenlogic: a quantum view for multiple-valued and fuzzy systems, in Quantum interaction, QI 2016, LNCS (Springer, Berlin, 2017), Vol. 10106, pp. 239-251

5. H. Haken, Synergetics (Springer, Berlin, 1977)

6. M. Lax, W. Cai, M. Xu et al., Random processes in physics and finance (Oxford University Press, Oxford, 2006)

7. A. Khrennikov, Information dynamics in cognitive, psychological, social, and anomalous phenomena, in Fundamental Theories of Physics (Kluwer, Dordrecht, 2004)

8. A. Khrennikov, Ubiquitous quantum structure (Springer, Berlin, 2010)

9. J. Busemeyer, P. Bruza, Quantum models of cognition and decision (Cambridge University Press, Cambridge, 2012)

10. E. Haven, A. Khrennikov, Quantum social science (Cambridge University Press, Cambridge, 2013)

11. M. Asano, A. Khrennikov, M. Ohya, Y. Tanaka, I. Yamato, Quantum adaptivity in biology: from genetics to cognition (Springer, Berlin, 2015)

12. E. Haven, A. Khrennikov (Eds.), The Palgrave handbook of quantum models in social science: applications and grand challenges (Palgrave Macmillan, Basingstoke, UK, 2017) 
13. E. Haven, A. Khrennikov, T.R. Robinson, Quantum methods in social science: a first course (WSP, Singapore, 2017)

14. A. Khrennikov, Found. Phys. 29, 1065 (1999)

15. A. Khrennikov, Biosystems 70, 211 (2003)

16. A. Khrennikov, Open Syst. Inform. Dyn. 11, 267 (2004)

17. M.E. Pothos, J.R. Busemeyer, Proc. R. Soc. B 276, 2171 (2009)

18. A. Brandenburger, Game Econ. Behav. 69, 175 (2010)

19. E.M. Pothos, J.R. Busemeyer, Behav. Brain Sci. 36, 255 (2013)

20. A. Khrennikov, I. Basieva, E.N. Dzhafarov, J.R. Busemeyer, PLoS One 9, e110909 (2014)

21. T. Boyer-Kassem, S. Duchene, E. Guerci, Theory Decis. 10, 1 (2015)

22. M. Asano, I. Basieva, A. Khrennikov, M. Ohya, Y. Tanaka, J. Math. Psychol. 78, 2 (2017)

23. T. Takahashi, S.-J. Kim, M. Narusec, Prog. Biophys. Mol. Biol. 130, 103 (2017)

24. R. Penrose, The Emperor's new mind (Oxford University Press, New York, 1989)

25. S. Hameroff, J. Conscious. Stud. 1, 91 (1994)

26. C. Brukner, A. Zeilinger, Phys. Rev. Lett. 83, 3354 (1999)

27. G.-M. D'Ariano, AIP Conf. Proc. 889, 79 (2007)

28. A. Plotnitsky, Proc. Conf. Quantum Theory 2, 309 (2002)

29. C.A. Fuchs, R. Schack, Found. Phys. 41, 345 (2011)

30. J. von Neumann, O. Morgenstern, Theory of games and economic behaviour (Princeton University Press, Princeton, NJ, 1944)

31. A. Tversky, D. Kahneman, Science 185, 1124 (1974)

32. D. Tversky, D. Kahneman, J. Risk Uncertain. 5, 297 (1992)

33. H. Haken, Light: vol. II: laser light dynamics (North-Holland Physics Publishing, Amsterdamn, 1985)

34. M. Fox, Quantum optics: an introduction (Oxford University Press, Oxford, 2006)

35. R. Hanbury Brown, R.Q. Twiss, Nature 177, 27 (1956)

36. C.K. Hong, Z.Y. Ou, L. Mandel, Phys. Rev. Lett. 59, 2044 (1987)

37. H.M. Wiseman, Phys Scripta 91, 033001

38. W. Elsäßer, arXiv:1607.03647

39. Z. Toffano, IEEE J. Sel. Top. Quantum Electron. 3, 485 (1997)

40. A.L. Schawlow, C.H. Townes, Phys. Rev. 112, 1940 (1958)

41. A. Orszag, in Les Lasers, principes, réalisations, applications (Masson et Cie, Paris, 1968), Chap. V

42. F. Bagarello, E. Haven, Phys. Scr. 90, 015203 (2015)

43. P. Khrennikova, J. Math. Psychol., 78, 76 (2017)

44. P. Barberá, J.T. Jost, J. Nagler, J.A. Tucker, R. Bonneau, Psychol. Sci. 26, 1531 (2015)

45. A. Smith, An inquiry into the nature and causes of the Wealth of Nations (1776), (reprinted) (University of Chicago Press, Chicago, 1977)

46. B. Falkenburg, Poznan Stud. Philos. Sci. Humanit 96, 207 (2008)

47. C.Z. Ning, IEEE J. Sel. Top. Quantum Electron. 19, 6516933 (2013)

48. T. Erneux, P. Glorieux, Laser dynamics (Cambridge University Press, Cambridge, 2010)

49. S. Grauwin, E. Bertin, R. Lemoy, P. Jensen, Proc. Natl. Am. Sci. USA 106, 20622 (2009)

50. C. Jaeger, G. Horn, T. Lux, D. Fricke, S. Frurst, W. Lass, L. Lin, A. Mandel, F. Meissner, S. Schreiber, D. Vesper, R. Zwiener, From the financial crisis to sustainability, Potsdam, European Climate Forum, 2009. www.european-climate-forum.net/ index.php?id=ecfreports

51. R. Collins, Interaction ritual chains (Princeton University Press, Princeton, 2004) 